\title{
Interference between gait and cognitive tasks in a rehabilitating neurological population
}

\author{
Patrick Haggard, Janet Cockburn, Josephine Cock, Claire Fordham, Derick Wade
}

\begin{abstract}
Objectives-To quantify the extent of interference between gait and cognitive tasks after brain injury; to investigate whether such interference is common to various cognitive tasks, or confined to specific cognitive modules; to investigate whether such interference declines during recovery from brain injury.

Method-Fifty participants were recruited from a neurological rehabilitation unit ( 33 people, $75 \%$ of sample); the stroke rehabilitation ward of an acute hospital (11 people, 20\%); and a young disabled unit (six people, $5 \%$ ). Measures of stride duration were taken in single task conditions, and in conjunction with each of four cognitive tasks. Outcome measures were dual task decrements in gait and in cognitive task performance.
\end{abstract}

Results-Overall, a $7 \%$ decrement in stride duration was recorded under dual task conditions compared with single task, with stride duration being significantly longer during simultaneous performance of each cognitive task. There was a $4 \%$ decrement on average in cognitive task performance under dual task conditions, with significant decrements being recorded for word generation while walking and paired associate monitoring while walking. A significant correlation $(r=0.45)$ was found between dual task decrements and scores on a standard measure of disability - the Barthel activities of daily living scale-but the correlation with $10 \mathrm{~m}$ walking time was not significant $(r=0.18)$. Conclusion-Interference between cognitive tasks and motor control activities such as gait is a problem in neurological rehabilitation settings. Interference between cognition and locomotor tasks may be important in assessing neurological patients' ability to function independently, and in designing therapies for both cognitive and motor rehabilitation.

(F Neurol Neurosurg Psychiatry 2000;69:479-486)

Keywords: motor control; dual task interference; neurological rehabilitation

In normal circumstances, people are able to perform motor tasks and higher cognitive functions at the same time. Thus, for example, most healthy adults can walk while holding a conversation. This ability contrasts with the classic psychological view of cognition as a limited capacity general purpose information transmission channel. ${ }^{1}$ The term "attention" has often been used to describe this central capacity. A strong version of this view predicts that people should have great difficulty doing any two tasks at once. A more moderate view holds that two tasks will interfere if they both use a common processing component. ${ }^{2}$ Indeed, concurrent performance of two cognitive tasks, such as reading while monitoring a conversation, often leads to a deterioration, or "dual task decrement", in the performance of either or both tasks. Motor tasks such as walking are sometimes said to be immune from this interference because they are "automatic". On a limited capacity view, this would mean that movement control does not require central cognitive resources. ${ }^{2}$ The limited capacity view has recently been challenged by a more modular view, in which distinct cognitive modules, localised in specific brain areas, perform specific processing operations. ${ }^{3}$ On this view, tasks which use non-overlapping sets of cognitive modules can be performed concurrently without dual task decrement. ${ }^{4}$

After acquired brain injury, however, the availability and use of different modules, such as those subserving cognition and movement, may be quite different from that in healthy people. Clinical observation provides examples of cases in which a patient can perform a cognitive task in isolation, and a motor task in isolation, but during concurrent performance one or both is severely impaired. Similar patterns of interference have been described in healthy elderly people. ${ }^{5620}$

This increased interference can be explained in at least two ways. Firstly, overall cognitive capacity may decrease after brain damage. For example, impairments of "attention" have been reported after stroke $^{78}$ and head injury. ${ }^{9-11}$ These impairments are typically seen on tasks involving rapid processing of large quantities of information, especially when tasks are externally paced. If brain damage reduces informational capacity, impairments should be common to all tasks. Such a view does not fit well with the common clinical experience that rehabilitating patients may function normally in some domains and in some tasks, while being severely impaired on others.

Secondly, cognitive motor interference may arise because motor control ceases to be automatic after acquired brain injury. The motor relearning tradition in rehabilitation is based on this premise. ${ }^{12}$ Damage to the neural structures of the motor system, or to the skeletomuscular system, may make a prelearned pattern of motor commands useless: a new pattern must be established. Thus, previously automatic actions may revert to the status of "controlled" processes and may place heavy demands on available cognitive resources. ${ }^{13}$ In 
the earlier stages of recovery, performing a motor task may only be possible if the full computational resources of central cognitive capacity are available to control the movement. This would mean that the additional capacity available for any concurrent cognitive task is diminished, producing a dual task cognitive decrement. Conversely, performance on the cognitive task may be preserved by diverting cognitive resources from the motor task, producing a dual task motor decrement. On this view, then, competition for limited central capacity between the two tasks leads to the dual task decrement in patients with brain injury. This has implications for clinical assessment: the degree of interference between motor and other concurrent tasks may be a potent indicator of the functional state of the motor system during rehabilitation. However, there has been little systematic evaluation of the extent to which motor control in rehabilitating patients may be compromised by competing attentional loads of other ongoing activities or may, in turn, compromise performance of other ongoing activities.

Cognitive motor interference may be of considerable clinical importance for several additional reasons. Firstly, a typical therapy regime itself involves concurrent performance of cognitive and motor tasks (for example, walking while attending to therapist's instructions). Poor dual task performance may therefore complicate therapy treatment. Patients may benefit either from therapies which minimise the requirement of dual task performance, or from therapies specifically selected to improve levels of dual task performance. Secondly, the level of dual task interference, and the precise conditions and task combinations under which it occurs, may vary between patients. Therefore, assessment and monitoring of a person's dual task performance could contribute to informed goal setting and treatment planning. Finally, because many everyday activities involve concurrent cognitive and motor components, a motor task performed under dual task conditions may provide a better index of functional everyday ability than a motor task performed under the single task conditions of typical neurological assessment. Understanding the nature, prevalence, and prognosis of dual task decrements could therefore form an important part of assessment.and rehabilitation.

This study therefore investigated cognitive motor interference with the following aims: to quantify the degree and severity of dual task cognitive-motor interference in a typical rehabilitating population; to investigate whether interference has a modular or a general pattern across different task combinations; to investigate its relation to site of brain lesion; and to investigate its course during the rehabilitation experience. Our study focused on walking, which is a major goal of many rehabilitation programmes, and is an integral part of many activities of daily living.
Table 1 Patient characteristics

\begin{tabular}{lllll}
\hline Variable & Mean & SD & Min & Max \\
\hline Age (y) & 50.18 & 16.47 & 18 & 84 \\
Time postonset (months) & 16.36 & 30.77 & 1 & 156 \\
Barthel scale score & 16.54 & 3.71 & 6 & 25 \\
10 m walk (s) & 28.84 & 40.01 & 5 & 250 \\
Men 28; women 22. & & & & \\
Aetiology: & & & \\
$\quad$ Right hemispheric stroke & 22 & & \\
$\quad$ Left hemispheric stroke & 8 & & \\
Brain stem stroke & 3 & & \\
$\quad$ SAH & 5 & & \\
$\quad$ Head injury & & 7 & & \\
$\quad$ Other & & 5 & & \\
Control subjects: & 45.30 & 17.75 & 23 & 75 \\
$\quad$ Age (y) & & & & \\
\hline
\end{tabular}

SAH=Subarachnoid haemorrhage.

Other=Anoxia (two), tumour removal (two), surgery for epilepsy (one).

\section{Methods}

Participants were selected from the patients at three neurological treatment units in the Oxford area: Rivermead Rehabilitation Centre for adults with acquired non-progressive brain injury, Ritchie Russell House Young Disabled Unit, and the stroke rehabilitation ward of the Radcliffe Infirmary. The sample covered the range of diseases typically encountered in neurological rehabilitation, and is described in more detail in table 1 . Inclusion criteria were an ability to walk $10 \mathrm{~m}$ without assistance of another person, adequate language comprehension and production to follow instructions given, written informed consent, and referral by medical staff. Fifty patients were studied over a 2 year period. Of these, 16 were seen on a second occasion to investigate longitudinal change in dual task performance. These were a subset of patients from the main sample who showed substantial dual task decrements in gait $(n=14)$ or in the cognitive tasks $(n=2)$. Finally, 10 healthy controls, from the same age range as the patients, were also studied.

The study received ethical approval from the nursing and allied professions research committee, Oxford (NAPREC).

\section{GAIT MEASUREMENT}

Unobtrusive pressure pads (Force Sensing Resistors $^{\mathrm{TM}}$ ) were taped to the ball and heel of each foot. The pressure signal on each pad was digitised on a laptop computer and stored for subsequent analysis. To analyse the gait signal, pressure traces from all four pads were subsequently displayed on a computer screen, and cursors were placed interactively to mark the start and end of stance phase (onset and offset of pressure on the heel and ball of each foot respectively). The number of strides, median duration, and variability (SD) in duration of the stride time (time from the onset of the right foot stance phase to the next onset of right foot stance phase) were calculated for each task performance.

This system of gait measurement has the advantage of technological simplicity, relatively non-invasive use, low cost, and flexibility. No special walking track or laboratory space is required, and the measurement equipment is portable. However, the system is unusual in that it measures only stride duration, and does 
not directly measure stride length or walking speed. A change in stride duration will, however, inevitably alter walking speed unless compensatory adjustments of stride length occur. Observation during testing suggested that dramatic changes in stride length did not occur during dual task performance, but subtle changes cannot be ruled out by our data. As successful timing of gait patterns is of fundamental importance in motor control, and because of the technological simplicity of our approach, we nevertheless considered ourselves justified in using a timing based measure of gait, rather than a direct measure of walking speed.

\section{COGNITIVE TASKS}

Stimulus materials for cognitive tasks were delivered over stereo headphones worn by the subjects. The stimuli were delivered by a laptop computer. Both stimulus and verbal response waveforms in the cognitive tasks were recorded through a compact microphone, digitised at 3 $\mathrm{kHz}$ in synchrony with the gait data, and stored on a laptop computer. Subsequent analysis involved interactive display of the stimulus and response auditory waveforms, and use of a visual cursor to mark onsets of stimulus and response events.

Four cognitive tasks were selected on the basis of pilot data, of previous reports in the literature, and of theoretical considerations regarding independent modules for specific cognitive processes. The tasks were as follows.

A spoken word generation task. ${ }^{14}$

Subjects were given a category ("things to eat", "things to drink", "things in the house" or "things in the street") and asked to generate as many exemplars of the category as possible until they heard the signal to stop (after 1 minute). The onset latency for each item, the number of correct items, the number of repetitions, and the number of non-items (for example, "um") were measured.

\section{A mental arithmetic task with auditory presentation $^{14}$}

Simple sums (for example, " $5+6=11$ ") were presented every 5 seconds. Subjects responded as quickly and as accurately as possible either "yes" or "no" to indicate whether each sum was correct or not. Responses and response latency from the start of each sum were recorded. This task was used for the first 32 patients studied only. At the end of the first year the mental arithmetic measure was discontinued, because it seemed relatively insensitive to interference from concurrent gait, because it made the length of the test battery impractical, and because overlap between the neural process for gait and for mental arithmetic is not clearly supported by psychological theory. For these reasons, a strategic decision was taken to drop this task from the test battery on the grounds that it did not provide additional insights into CMI.
$A$ verbal paired associate monitoring task $k^{16}$

Subjects were first asked to remember a pair of associated words (for example, "dog", "bone"). They were then presented with 40 words, occurring every 1.5 seconds. The target pair occurred 6 times within the list. Subjects were asked to respond "yes" only when the target pair was heard in the correct order. The stimulus list included lures in which individual items of the target pair occurred (for example, "dog", "biscuit") but not the full target pair. The number of target pairs correctly detected, and the median latency of correct responses were measured.

\section{$A$ visuospatial decision task $k^{14}$}

Subjects heard speech segments describing times of day (for example, " 10 past 3", "25 to 7"), presented every 5 seconds. Subjects responded as quickly and as accurately as possible whether the hour and minute hands would be on the same side or on different sides of the clock face, for each time given. Responses and response latency from the start of each time were recorded.

Subjects were given initial examples and introductory training before each new cognitive task. Four versions of each cognitive task were prepared, using different stimulus lists or category names as appropriate. The versions were equated for task difficulty in informal testing.

Other measures taken were $10 \mathrm{~m}$ walking time $^{17}$ and a version of the Barthel activities of daily living (ADL) scale to provide an overall measure of disability. ${ }^{18}$ The Barthel ADL is a well established and validated scale widely used to measure functional ability in rehabilitating populations.

\section{PROCEDURE}

Each testing session involved single task recording of gait and of cognitive task performance, and also dual task recording of both tasks combined. In single task walking trials, the subject walked continuously at a comfortable pace in the gymnasium or ward setting. Subjects used any walking aid (stick, frame) that they were used to. A healthcare professional walked with the subject in case of falling, but did not assist them during testing. A second experimenter followed behind the subject carrying the data acquisition equipment. Data acquisition started after the subject had begun to walk and continued for 60 seconds. Subjects turned round when they reached the end of the gymnasium or ward. Alhough the number of turns per trial was not specifically recorded by our equipment, it was typically between 0 and 3 .

In single task cognitive trials, subjects performed one of the four cognitive tasks described above while sitting either in a wheelchair or on a physiotherapy plinth. The duration of each cognitive task was 60 seconds.

In dual task trials, the subjects performed both walking and the cognitive tasks simultaneously. Subjects began walking, and the experimenter then initiated one of the four cognitive tasks once walking was under way. Gait signals 
Table 2 Median stride duration (ms)

\begin{tabular}{lllll}
\hline Group & Cognitive task & $\begin{array}{l}\text { Single task } \\
\text { Mean }(S D)\end{array}$ & $\begin{array}{l}\text { Dual task } \\
\text { Mean }(S D)\end{array}$ & \% Decrement \\
\hline $\begin{array}{llll}\text { Patients (n=50) } \\
(\mathrm{n}=32)\end{array}$ & Word generation & $1238(725)$ & $1325(800)$ & $7^{\star \star}$ \\
& Mental arithmetic & $1366(629)$ & $1472(786)$ & $8^{\star}$ \\
& Paired associate monitoring & $1261(770)$ & $1334(866)$ & $6^{\star \star}$ \\
Controls (n=9) & Visuospatial decision & $1208(754)$ & $1293(810)$ & $7^{\star \star}$ \\
$(\mathrm{n}=10)$ & Word generation & $579(117)$ & $634(166)$ & $9\left({ }^{\star}\right)$ \\
$(\mathrm{n}=10)$ & Paired associate monitoring & $578(122)$ & $570(140)$ & -1 \\
& Visuospatial decision & $519(116)$ & $504(151)$ & -3
\end{tabular}

$\left.{ }^{\star}\right) \mathrm{p}<0.10 ;{ }^{\star} \mathrm{p}<0.05 ;{ }^{\star \star} \mathrm{p}<0.01$

Control scores are not reported for stride duration during mental arithmetic because only three controls performed this task. A negative \% decrement indicates better performance under dual task conditions. dual task gait decrements significant at the 5\% level. This is well above the proportion expected by chance (binomial $(n=50, p=0.05$, $x \geqslant 20): p<0.001)$. Of these 20 , nine had a right hemispheric stroke, four a left hemispheric stroke, one a brain stem stroke, two a subarachnoid haemorrhage, two a head injury, and two had other diagnoses. Comparison of these figures with table 1 shows that dual task decrement was distributed relatively evenly across pathological groups in our sample. Two patients showed significant dual task increments, as might be expected by chance (binomial $(n=50, p=0.05, x \leqslant 2): p=0.54)$.

The overall analysis was followed by separate ANOVAs conducted on gait data collected during each cognitive task (table 2). The dual task gait decrement was statistically significant for all four cognitive tasks, with a percentage decrement of $7 \%$ for category generation, $8 \%$ for auditory mental arithmetic $(n=32), 6 \%$ for paired associated monitoring, and $7 \%$ for visuospatial decision.

Healthy controls

The healthy controls showed no significant dual task decrement on the paired word associate and visuospatial judgement tasks (all $\mathrm{p}>0.27$ ), but showed a borderline significant dual task decrement of $59 \mathrm{~ms}$ on the word generation task $(F(1,8)=5.24, \mathrm{p}=0.051)$ (one subject did not comply with instructions, and was excluded from this analysis).

\section{Effects of lesion site}

We next investigated effects of lesion site on dual task decrement in the subsample of patients with a cerebrovascular accident with left $(n=8)$ and right $(n=22)$ sided lesions reported on CT, for whom no data were missing. Patients with other diagnoses were excluded from this analysis, as the lesion site was typically either unknown or multiple. In none of our tasks did the lateralisation of the lesion significantly affect the dual task decrement in stride duration of these patients (all $\mathrm{p}>0.14$ ). We further analyzed the durations of the left and right swing phases of gait in these patients. This analysis showed that the dual task decrement in swing duration did not differ significantly between the foot ipsilateral and the foot contralateral to the lesion $(p=0.391)$.

\section{Variability of gait cycle}

Median stride durations and variability in duration of stride time are shown in table 2 .

\section{Patients}

Analysis of variance was applied to the median stride duration of the 50 patients. Task context (single or dual) was a within subjects factor. Pooling across all four cognitive tasks, stride duration was significantly greater in the dual task condition (mean 1333 (SD 810) ms) than in the single task condition (mean 1244 (SD $758) \mathrm{ms}): \quad F(1,49)=16.13, \mathrm{p}<0.001$. This represents a dual task gait decrement of $7 \%$. Separate analysis of individual subjects' data, using repeated trials as a random factor, disclosed that 20 of the 50 patients showed
We analysed the SD of the durations of the strides made in each trial, to assess whether the variability of gait, rather than its median duration, was affected by dual task performance. No concurrent cognitive task caused a significant change in the variability of gait timing (all $\mathrm{p}>0.20$ ).

\section{COGNITIVE TASK EFFECTS}

The four cognitive tasks do not share a common scoring system. Further, each task was replicated only twice in single task and twice in dual task context. Therefore, it is not possible to pool results across tasks, or to calculate significance levels for dual task cognitive decrements in individual subjects. No cog- 
Table 3 Cognitive task decrement: patients $(n=50)$. Cognitive task performance: control subjects $(n=10)$

\begin{tabular}{|c|c|c|c|c|c|}
\hline Task & $\begin{array}{l}\text { Single } \\
\text { Mean (SD) }\end{array}$ & $\begin{array}{l}\text { Dual } \\
\text { Mean (SD) }\end{array}$ & $t$ Value & $p$ Value & $\%$ Decrement \\
\hline \multicolumn{6}{|c|}{ Cognitive task decrement: patients $(n=50)$} \\
\hline Word generation: & & & & & \\
\hline Accuracy & $16.11(5.37)$ & $14.87(5.36)$ & 2.26 & $\star$ & 6 \\
\hline Mental arithmetic $(n=32)$ : & & & & & \\
\hline Accuracy $(\max =12)$ & $10.55(1.63)$ & $10.17(2.12)$ & 1.62 & NS & 4 \\
\hline Paired associate monitoring: & & & & & \\
\hline Accuracy $(\max =6)$ & $5.13(0.95)$ & $4.83(1.21)$ & 2.32 & * & 5 \\
\hline Visuospatial decision: & & & & & \\
\hline Accuracy $(\max =12)$ & $9.57(2.40)$ & $9.23(2.52)$ & 1.95 & $\left({ }^{\star}\right)$ & 2 \\
\hline \multicolumn{6}{|c|}{ Cognitive task performance: control subjects $(n=10)$} \\
\hline $\begin{array}{l}\text { Word generation }(n=9) \text { : } \\
\text { Accuracy }\end{array}$ & $24.78(2.76)$ & $24.50(4.30)$ & 0.26 & NS & 1 \\
\hline Paired associate monitoring: & & & & & \\
\hline Accuracy & $5.75(0.42)$ & $5.65(0.41)$ & 0.80 & NS & 1.5 \\
\hline Visuospatial decision: & & & & & \\
\hline Accuracy & $11.78(0.66)$ & $11.78(0.36)$ & & NS & 0 \\
\hline
\end{tabular}

$\left({ }^{\star}\right) \mathrm{p}<0.10 ;{ }^{\star} \mathrm{p}<0.05$.

Control scores are not reported for mental arithmetic because only three controls performed this task.

nitive task showed a significant difference in median response latency between single and dual task conditions. Mean scores for each cognitive task are shown in table 3.

\section{Patients}

The mean number of correct items in single task word generation (16.1 (SD 5.37)) was significantly higher than the mean number in the dual task condition (14.9 (SD 5.36): $t(49)=2.26, \mathrm{p}<0.03$. Further, the number of non-items (for example, "um") was significantly lower in the single task condition (2.94 (SD 3.39)) than in the dual task condition (3.94 (SD 3.80)): $t(49)=3.68, \mathrm{p}<0.001$. Repetitions of previously generated correct items were less common in the single task condition (1.06 (SD 1.10)) than in the dual task condition $(1.65$ (SD 1.34)): $t(49)=2.98$, $\mathrm{p}<0.01$.

We then subdivided the word generation score into the number of valid items in the first 30 seconds and the last 30 seconds of each trial. This analysis showed that the significant dual task decrement was confined to the last 30 seconds of each trial (mean decrement $=0.93$, $t(49)=2.58, \mathrm{p}<0.02$ ), with only minimal decrement in the first 30 seconds (mean $=0.14$, $t(49)=0.433, \mathrm{p}=0.67)$. Repetitions and nonwords were less marked in the first $30 \mathrm{~s}$ of each trial (repetitions: $t(49)=-1.80, \mathrm{p}<0.08$; nonwords: $\mathrm{t}(49)=-2.21, \mathrm{p}<0.04)$ than in the last 30 seconds (repetitions: $t(49)=-3.24, \mathrm{p}<0.01$; non-words: $t(49)=-3.20, \mathrm{p}=0.01)$.

There was no significant effect of task context on the auditory mental arithmetic score for the subset $(n=32)$ who did this task (single task mean score 10.55 (SD 5.37); dual task 10.17 (SD 2.12)), but the number of incorrect responses was significantly lower in the single task condition (0.61 (SD 0.69)) than in the dual task condition (1.00 (SD 0.97): $t(31)=2.52, \mathrm{p}<0.02$.

In the paired associate monitoring task, the mean number of target pairs correctly detected was significantly higher in the single task condition (5.13 (SD 0.95) than in the dual task condition (4.83 (SD 1.21)): $t(48)=2.32$, $\mathrm{p}<0.03$.
In the visuospatial decision task there was a trend towards a greater number of correct responses in single task conditions (9.57 (SD 2.40)) than in dual task conditions (9.23 (SD 2.52)): $t(49)=1.95, \mathrm{p}<0.06$.

Healthy controls

Control subjects did not show a significant difference between dual and single task score on any cognitive task. Moreover, unlike the patients, control subjects did not show a significant increase in dual task interference in the second 30 second period of the word generation task.

\section{Effects of lesion site}

We investigated effects of lesion site on dual task cognitive decrement in the subsample of patients with a cerebrovascular accident with left $(n=8)$ and right $(n=22)$ lesions (see gait analysis above). None of the cognitive task measures showed interactions between task context (single $v$ dual) and lesion site in ANOVA (all $\mathrm{p}>0.09$ ).

REGRESSION ANALYSES

To investigate the relation between dual task gait decrement and clinical profile, we calculated for each patient an average of the percentage decrements in median stride duration across all the cognitive tasks. We then performed a multiple partial regression to predict the dual task gait decrement from patient age, months since onset, Barthel ADL score, and $10 \mathrm{~m}$ walking time. The only significant predictor was Barthel ADL score $(t(44)=-2.40, \quad \mathrm{p}<0.03)$, which correlated $(r=-0.45)$ with dual task gait decrement. Interestingly, the $10 \mathrm{~m}$ time, a widely used clinical measure of locomotor function, correlated only very weakly with dual task gait decrement $(r=0.18)$. Moreover, the association between $10 \mathrm{~m}$ time and ADL score was weaker than the association between dual task decrement and ADL score. In this sense, dual task decrement was quite closely related to patients' functional independence, whereas standard gait measures were not.

MICROSTRUCTURAL ANALYSIS OF INTERFERENCE We investigated short term interference between gait and cognitive tasks by looking for time sharing between gait and word generation. A time sharing approach combines tasks by alternating the allocation of limited cognitive resources between them. Word generation is self paced, unlike the other cognitive tasks studied here, and is therefore the most amenable to time sharing. Accordingly, we calculated the number of right footfalls that occurred in the 1 second before the patient's vocal onsets in word generation, and in the 1 second after vocal onset. Patients made significantly more steps in the 1 second window preceding the vocal onset (mean 3.9 (SD 3.1)) than in the 1 second window after vocal onsets (mean 3.5 $(\mathrm{SD} 2.9)): F(1,44)=4.33, \mathrm{p}<0.023$. This finding suggests a reduction in gait activity during and just after responding in word generation. 
Two tasks which relied on independent information processing operations should not show such systematic timing variations.

LONGITUDINAL STUDY

We compared the dual task decrement in stride duration between the first and second testing occasions of the 16 patients selected for longitudinal study. Although their mean time postonset was shorter than for the group as a whole (7 months) they did not differ in any other demographic respect. Too few longitudinal patients performed the mental arithmetic task for statistical analysis, so the results are given for the remaining three cognitive tasks. The mean interval between testing occasions was 181 (SD 81) days. A factorial analysis was used with testing occasion (first, second) and task context (single, dual) as factors. A significant interaction would imply longitudinal change in the dual task decrement. Significant main effects of testing occasion were found in all three tasks analyzed, due to reductions in overall stride duration of $193 \mathrm{~ms}$ (category generation), $207 \mathrm{~ms}$ (paired associates), and $220 \mathrm{~ms}$ (visuospatial decision). For category generation and for paired associates the dual task gait decrement did not change significantly between the first and second testing occasions $(\mathrm{p}=0.28$ and $\mathrm{p}=0.91$ respectively). The dual task decrement for visuospatial decision reduced from $76 \mathrm{~ms}$ at first testing to 24 $\mathrm{ms}$ at second testing, producing a significant interaction $(F(1,11)=5.19, \mathrm{p}<0.05)$ (data were incomplete for four longitudinal patients in this task). Nevertheless, longitudinal changes in dual task decrement were numerically smaller, on average, and statistically less remarkable than overall longitudinal changes in single task performance.

Similar factorial analyses of the cognitive data showed no significant main effects of testing occasion (all $\mathrm{p}>0.22$ ), and no significant interactions with task context (all $p>0.42$ ) for any of the three cognitive tasks. Analyses of individual longitudinal change in gait and in cognitive task performance will be the focus of a separate paper.

\section{Discussion}

Interference between cognition and gait was found after brain injury. This interference produced significant impairment of both gait and cognitive function in patients. In healthy controls, by contrast, dual task decrements in both gait and in cognitive scores were generally small or absent, and achieved borderline significance only for gait measures during word generation. Dual task decrement was not strongly related to lesion site. Decrements did not differ dramatically across the four cognitive tasks studied here. The gait decrement persisted in a subsample of patients studied longitudinally, despite significant recovery of single task gait performance. Gait decrements were negatively correlated with patients' Barthel ADL scores but were not strongly related to $10 \mathrm{~m}$ walking time. Detailed analysis of decrements in self paced word generation showed that decrements increased as testing trials proceeded, and were greater during the 1 second after word onset than in the 1 second before.

Interference between motor control and cognitive tasks after brain injury has been reported previously in the literature. ${ }^{15}{ }^{19}$ It has also been reported in elderly adults. ${ }^{5619}$ Our study provides more quantitative information from a larger sample of patients, and a greater range of cognitive tests than previous studies in the rehabilitation context. Specifically, we found significant slowing of the gait cycle, and significant reduction in cognitive task scores when both tasks were performed simultaneously. The size of the dual task decrement $(7 \%$ in stride duration and $4 \%$ in cognitive scores) is comparable between this study and earlier reports in similar patient groups. ${ }^{15}$ However, our study differs from previous approaches in showing that both motor and cognitive function may be simultaneously impaired in dual task performance. As many activities of daily living, such as cooking, involve concurrent motor and cognitive components, we emphasise the importance of quantitative measurement of both tasks in producing a complete clinical picture of dual task interference.

Our study also differs from others in the literature in using a range of structured, quantitative cognitive tests. Many other studies have investigated interference between movement and a single cognitive task. Hartman et al ${ }^{19}$ studied a visuomotor tracking task under three different task conditions. Their conditions were chosen specifically to examine a hypothesis of central executive deficit after head injury and only one measure (digit span) was a standard cognitive test. Such studies, therefore, cannot disclose whether interference occurs because motor function in the patients competes for use of the same cognitive resource as a specific cognitive task, or because motor function requires increased general cognitive capacity in the patients, while being automatic in the controls. By contrast, our four cognitive tasks were designed specifically to engage identified cognitive modules: executive function in the category generation task, number manipulation in the arithmetical task, memory function in the paired associate task, and visuospatial representation in the clock decision task. We found that both gait and cognitive score decrements were broadly comparable across tasks.

Our result is, in principle, compatible with different subgroups of patients showing interference in each of the tasks. For example, right hemispheric damage might be expected to affect visuospatial processing and left hemispheric damage to affect numerical processing, ${ }^{21}$ such that patients with left or right stroke might be expected to show dual task cognitive decrements primarily on the task subserved by the damaged hemisphere. However, breakdown of both gait and cognitive data by lesion site did not show any consistent relation between lesion site, cognitive task, and dual task decrement in either cognitive or gait scores. Therefore, we did not find strong evidence in our data for a highly modular pat- 
tern of cognitive-motor interference. We could not identify specific cognitive modules or specific brain structures, the involvement of which was required for cognitive-motor interference. However, this is a null result, which must be interpreted cautiously, and does not disprove the possibility of modular patterns of cognitive-motor interference.

Our result contrasts with previous studies, which have reported interference between specific combinations of motor and cognitive tasks. For example, Haggard and Cockburn ${ }^{14}$ noted interference between visuomotor tracking and the visuospatial decision task used here, but not between tracking and a mental arithmetic task. The more generalised decrement in the present study may be due to the wide involvement of several processes in walking after brain injury. Our data, therefore, point towards a general cognitive capacity explanation of dual task interference between gait and the cognitive tasks studied here. Information processing psychology has modelled cognitive operation using a limited capacity informationtransmission channel. ${ }^{2}$ On this view, dual task performance would exceed available information capacity in the patients, but not in healthy controls.

Could an overall reduction in patients' information processing capacity, as opposed to increased involvement of central information processing in locomotion, equally explain our results? Such an overall reduction should produce impairment in patients' single task cognitive performance, as well as large dual task decrements in the patients. However, our data showed that patients scored close to control levels in single task performance on the paired associate and on the visuospatial decision tasks, yet, unlike controls, had significant dual task decrements on those tasks. Thus, our results seem to reflect increased use of central resources for locomotion after brain injury, rather than reduced information capacity in the patient group.

Interference affects information processing in both tasks: dual task performance alters the speed (gait decrement) and accuracy (cognitive scores) of information processing reciprocally. Hockey $^{23}$ has suggested that information capacity is not rigidly fixed, but can be temporarily increased by voluntary effort. We think that our analysis of the timing of word generation decrements agrees with this view. Our patients' dual task decrement was confined to the last 30 seconds of the task. During the first stages of dual task performance, then, patients may transiently have increased their processing capacity, but they were unable to maintain this.

We speculate that the concept of effort may explain why cognitive-motor interference is a frequent and important clinical observation, but produces quite small changes in performance in laboratory studies such as ours. Some studies have even reported reliable dual task increments. The patients may have marshalled extra cognitive capacity by special effort in response to the unusual testing situation. Such a response would reduce any interference effects. Laboratory tests of any type may there- fore underestimate the true extent of cognitivemotor interference, and hence its importance in daily living. Future research might profitably focus on more observational, ambulatory assessment of dual task interference

Our control group showed a trend towards increase in median stride duration during word generation that was not evident in the other tasks. This represented a large proportion of stride duration, and might have reached significance with a larger sample. Word generation is the only one of our tasks in which cognitive output is self paced. It seems that, when the option existed, at least some of our controls prioritised the cognitive element at the expense of the pattern of stride duration established when walking alone, despite absence of instruction to favour either task. As this result is at variance with other published findings, ${ }^{6}$ it needs further exploration with a larger sample.

Our study also has several clinical implications. Firstly, we think that it raises important questions for assessment of motor function. A patient who recovers to a level when they can walk adequately in a quiet gymnasium might not be safe walking in a more demanding environment, such as a shopping street, where more cognitive distractors are present, or an uneven slippery surface. Moreover, the longitudinal section of our study suggests that recovery of single task motor performance during rehabilitation may mask a more persistent difficulty with dual task situations. Difficulty with dual task performance may therefore go unnoticed. More generally, we suggest that motor assessment should take into account the cognitive context within which motor actions occur. Most daily living tasks involve concurrent movement and cognition, yet quantitative assessments after brain injury typically treat these functions separately. Further, retraining of one function rarely takes into account status in the other. We suggest that measures of dual task interference should be included in standard clinical assessment protocols and used to inform content of therapy programmes.

Our study also has important implications for therapy practice in rehabilitation settings. A physiotherapy session, for example, may involve the therapist instructing the patient during the course of a movement. The instructions could thus represent a cognitive load which would interfere with practice of the movement. At the same time, the practised movement might reciprocally interfere with the comprehension of instructions. Other conversations and stimuli may be present in the gymnasium at the same time, increasing cognitive load still further. The style of interaction with patients and the design of therapy sessions may benefit from a greater understanding of the effects of cognitive-motor interference.

This research was supported by National Health Service Executive Research and Development, grant PCD2/A1/282. We thank the staff of Rivermead Rehabilitation Centre, Ritchie Russell House, and Beeson Ward and the Day Hospital, Radcliffe Infirmary, Oxford, for their assistance with this Radcliffe Infirmary, Oxford, for their assistance with this
project. We also thank all the participants for their time and project. We als
cooperation. 
1 Broadbent DE. Perception and communication. Oxford: Pergamon Press, 1958.

2 Broadbent DE Task combination and selective intake of information. Acta Psychologica 1982;50:253-90.

3 Shallice TE Specific impairments of planning. Philos Tran $R$ Soc Lond B Biol Sci 1982;298:199-209.

4 Shallice TE, McLeod P, Lewis K. Isolating cognitive modules with the dual-task paradigm: are speech perception and production separate processes? $Q \mathcal{F}$ Exp Psychol $A$ 1985;41A:235-50.

5 Maylor EA, Wing AM. Age differences in postural stability are increased by additional cognitive demands. $\mathcal{F}$ Gerontol $B$ 1996;51:143-54.

6 Camicioli R, Howieson D, Lehman S, et al. Talking while walking: the effect of a dual task in aging and Alzheimer's disease. Neurology 1997;48:955-8.

7 Wade DT, Wood V, Hewer RL. Recovery of cognitive function soon after stroke: a study of visual neglect, attention span and verbal recall. $\mathcal{F}$ Neurol Neurosurg Psychiatry 1988 ; 51:10-13.

8 Marshal SC, Grinnell D, Heisel B, et al. Attentional deficits in stroke patients: a visual dual task experiment. Arch Phys Med Rehabil 1997;78:7-12.

Ponsford J, Kinsella G. Attentional deficits following closedhead injury. $\mathcal{F}$ Clin Exp Neuropsychol 1992;14:822-38.

10 Stablum F, Leonardi G, Mazzoldi M, et al. Attention and control deficits following closed head injury. Cortex 1994;30:603-18.

11 Spikman JM, van Zomeren AH, Deelman BG. Deficits of attention after closed-head injury: slowness only? $\mathcal{f}$ Clin Exp Neuropsychol 1996;18:755-67.

12 AL Canning, CG Carr, JH Kilbreath, et al. Task-specific training of reaching and manipulation. In: Bennett, Keree $\mathrm{MB}$, Castiello $\mathrm{U}$, eds. Insights into the reach to grasp movement. Advances in psychology. 1994;105:239-65. Amsterdam, Netherlands: Elsevier.
13 Mulder T, Nienhuis B, Pauwels J. Prediction of independent mobility following damage to the nervous system. In: Fries W, ed. Ambulante und Teilstationäre Rehabilitation von Hirnverletzen. Munich: W Zuckschwerdt Verlag, 1996:52-60.

14 Haggard P, Cockburn J. Dividing attention between cognitive and motor tasks in neurological rehabilitation. Neuropsychological Rehabilitation 1998;8:155-70.

15 Geurts AC, Ribbers GM, Knoop JA, et al. Identification of static and dynamic postural instability following traumatic brain injury. Arch Phys Med Rehabil 1996;77:639-44.

16 Cicerone KD. Clinical sensitivity of four measures of attention to mild traumatic brain injury. Clin Neuropsychol 1997; 11:266-72.

17 Wade DT. Measurement in neurological rehabilitation. Oxford: Oxford Medical Publications, 1992:78-79.

18 Collin C, Wade DT, Davies S, et al. The Barthel index: a reliability study. International Disability Studies 1988;10:613.

19 Hartman A, Pickering R, Wilson BA. Is there a central executive deficit after severe head injury? Clin Rehabil 1992;6:133-40

20 Lindenberger U, Marsiske M, Baltes P. Dual-task costs in sensorimotor and intellectual functioning: increase from early adulthood to old age. Psychol Aging (in press).

21 Lezak MD. Neuropsychological assessment. 3rd ed. New York: Oxford University Press, 1995:60-1.

22 Crawford JR, Moore JW, Cameron IM. Verbal fluency A NART-based equation for the estimation of premorbid performance. Br f Clin Psychol 1992;31:327-9.

23 Hockey GRJ. Compensatory control in the regulation of human performance under stress and high workload. A cognitive-energetical framework. Biol Psychol 1997;45:7393. 